wünschte Zahlenkombination wird direkt über das Kartenlesegerät eingegeben. Mit dem DGN PINtool, das im Internet gratis zur Verfügung steht, lässt sich zudem der Kartenstatus auslesen, d. h. ob die Karte aktiv ist oder gesperrt wurde.

Karteninhaber können über das DGN PINtool zum einen die Transport-PINs (d. h. produktionsseitig aufgebrachte PINs zur Absicherung des Kartentransports) brechen, um ihre Karte zu aktivieren. Zum anderen können sie die Signatur- und die Karten-PIN ändern bzw. mittels PUK entsperren.

Damit ist das neue Werkzeug eine Ergänzung des DGN Kartenprüftools, das bereits im Juni dieses Jahres online ging. Letzteres prüft detailliert, ob die Karte technisch einwandfrei ist und deren PINs auch wirklich funktionieren. Mit wenigen Klicks lässt sich zum Beispiel feststellen, ob eine PIN durch mehrfache Falscheingabe gesperrt oder noch gar nicht aktiviert wurde. In beiden Fällen ist die Signaturkarte nicht einsatzbereit.

Sowohl das DGN PINtool als auch das Kartenprüftool laufen aktuell unter Windows sowie Mac OS X und sind für Signaturkarten nutzbar, die im DGN Trustcenter produziert wurden. Dazu zählen: DGN sprintCard, DGN businessCard, medisign eArztausweis, der elektronische Ausweis für Zahnärzte (medisign ZOD Card 2.0), medisign ePsychotherapeutenausweis (ePTA) sowie medisignCard (2.0). Die Karten verfügen jeweils über zwei PINs - eine für die qualifizierte Signatur und die andere für alle anderen Kartenfunktionalitäten, u. a. Authentifizierung und Verschlüsselung. Vor dem ersten Einsatz müssen beide PINs einzeln aktiviert werden.

Das DGN PINtool und das Kartenprüftool stehen im Internet unter folgendem Link zur kostenlosen Nutzung bereit: www.dgn. de/produkte/hilfe-support/

\section{Open-Data-Apps für transparente Verwaltung}

Ein am 8.11.2011 gestarteter Wettbewerb für die Entwicklung von Apps will die Nutzung von frei zugänglichen Daten der öffentlichen Verwaltung fördern. Die drei Organisationen Government 2.0 Netzwerk Deutschland, Open Data Network und Open Knowledge Foundation Deutschland starteten ihre Initiative unter dem Motto "Apps für Deutschland" auf der Messe Moderner Staat in Berlin. Unterstützt wird der Wettbewerb vom Bundesinnenministerium, dem Statistischen Bundesamt, dem ITK-Verband Bitkom, dem Deutschen Städte- und Gemeindebund sowie Bremen und Berlin.

"Öffentliche Daten sind kein Schatz, der beschützt werden muss, sondern vielmehr ein Rohstoff, aus dem sich viele spannende und wichtige Anwendungen bauen lassen", erklärte Stefan Gehrke vom Open Data Network. Demokratische Prozesse erforderten aufgeklärte Bürger. Wer zum Beispiel wisse, wofür der Staat die Steuereinnahmen verwende oder Subventionen verteile, sei auch in der Lage, diese Prozesse kritisch zu begleiten.

Für Regierungen und Verwaltungen bedeute dies allerdings auch, "die Deutungshoheit über Informationen zu teilen“, gab Gehrke zu bedenken und fügte hinzu: „Diese Folge von Transparenz fällt oft noch schwer." Anke Domscheit-Berg vom Government 2.0 Netzwerk Deutschland erklärte in einer Pressemitteilung: „Open Government umfasst neben mehr Transparenz auch neue Formen der Kooperation von Bürgerlnnen und Staat auf Augenhöhe."

Der Wettbewerb ist in zwei Hälften geteilt: Bis zum 15. Dezember können Teilnehmer Ideen für die Nutzung offener Daten einreichen. Entwickler können ihre Web-Anwendungen oder Apps bis zum 1. Februar 2012 einreichen. Die Preise werden von einer Jury aus Wissenschaft, Verwaltung und Zivilgesellschaft vergeben und auf der CeBIT 2012 verliehen.

Weitere Informationen: http://apps4deutschland.de/

\section{Symantec-Studie zeigt sinkende Aufmerksamkeit für Cybergefahren}

Das Interesse an der Teilnahme an staatlichen Programmen zum Schutz kritischer Infrastrukturen ist in Unternehmen im Vergleich zu 2010 weltweit gesunken. In Deutschland wissen nur 34 Prozent der Firmen von derartigen Programmen. Dies ist das Ergebnis des am 08.11.2011 veröffentlichten der "Critical Infrastructure Protection (CIP) Survey 2011", bei der Symantec bereits zum zweiten Mal Unternehmen aus Schlüsselindustrien wie Banken oder der Energieversorgung befragte. Hinzu kommt, dass Unternehmen weniger Ressourcen für die Sicherheit ihrer IT bereitstellen. Dies ist besonders beunruhigend, da ein Cyberangriff auf Unternehmen mit kritischen Infrastrukturen weitreichende Folgen für die nationale Sicherheit haben kann.

Attacken wie Nitro und Duqu zielen auf Schwachstellen in kritischen Infrastrukturen ab. Vor diesem Hintergrund beunruhigen die Ergebnisse der neuen Symantec-Studie. Sie untersuchte, wie Betreiber von Netzwerken, die für die Wirtschaft und Gesellschaft als kritisch gelten, ihre aktuelle Sicherheitslage einschätzen.

In diesem Jahr nahmen deutlich weniger Unternehmen an einem staatlichen Schutzprogramm teil. Das überrascht kaum, da die Betreiber ihre Anstrengungen zum Schutz kritischer Netze wegen Personal- und Budgetmangel stark einschränken müssen. Sie konzentrieren sich primär auf aktuelle Bedrohungen und vernachlässigen dadurch weitreichendere Schutzmaßnahmen. Dies ist eine besorgniserregende Entwicklung, stehen doch Betreiber kritischer Infrastrukturen mehr denn je im Visier organisierter Cyberkrimineller. Unternehmen und Regierungen weltweit sollten den Schutz dieser für Wirtschaft und Gesellschaft wichtigen Netzwerke stärker forcieren und in das Zentrum ihrer Bemühungen stellen.

Um der steigenden Bedrohung Rechnung zu tragen, erweiterte Symantec den Kreis der befragten Sektoren. Während 2010 nur sechs Bereiche untersucht wurden, nimmt der CIP 2011 inzwischen 14 Sektoren unter die Lupe. Dazu zählen das Energie-, Banken- Versicherungs- und Finanzwesen sowie Sektoren wie Telekommunikation, IT, Gesundheit, der öffentliche Dienst, Informationstechnologie, Landwirtschaft, Regierungen, Fertigungsunternehmen, der Nah- und Fernverkehr sowie öffentliche Bau- und Chemiefirmen.

Die wichtigsten Ergebnisse der Studie auf einen Blick: Geringes Engagement und Interesse an staatlichen Schutzprogrammen

Während 2010 die CIP-Programme der Regierungen noch gut angenommen wurden, sank 2011 das Interesse der Unternehmen. Gerade einmal 36 Prozent der weltweit Befragten wussten überhaupt von den Regierungsplänen zum Schutz kritischer Infrastrukturen oder hatten sich zumindest teilweise um Einblick bemüht. Im vergangenen Jahr waren es immerhin 55 Prozent. In Deutschland sieht die Situation ähnlich aus: Lediglich 34 Prozent waren staatliche CIP-Programme bekannt. So ging auch die aktive Beteiligung daran zurück: 2010 nahmen weltweit noch 56 Prozent der Studienteilnehmer vollständig oder teilweise an den Programmen teil. In 